Check for updates

Cite this: Nanoscale Adv., 2019, 1, 602

\title{
Configuration- and concentration-dependent hybrid white light generation using red, green, and blue quantum dots embedded in DNA thin films
}

\author{
Velu Arasu, ${ }^{a}$ Deoksu Jo, ${ }^{a b}$ Heeyeop Chae, (iD *ab Ho Kyoon Chung*a \\ and Sung Ha Park (iD *ac
}

Artificial white-light production from primary red, green, and blue (RGB) colours is in high demand in the lighting, information technology, and wearable display industries, and it requires a simple device structure and efficient templates with stable luminophores. To realize efficient and stable optoelectronic devices, relevant materials and device structures need to be identified. Therefore, we report the construction of a simple hybrid whitelight optoelectronic device with a single excitation source with efficient RGB colours on a stable optical platform. Emission wavelength-tunable R, G, and B quantum dots (QDs) with specific ligands and cetyltrimethylammonium chloride (CTMA)-modified DNA (CDNA) are prepared for the fabrication of QDs embedded in CDNA thin films with mixed and orthogonally stacked configurations. Fourier transform infrared, photoluminescence quantum yield (PLQY), ultraviolet (UV)-vis absorbance, photoluminescence, and electroluminescence (excited by blue and UV LEDs) spectra of the QDs embedded in CDNA thin films are analyzed to investigate their ligand attachment, luminescence efficiency, optical excitation, spectral emission, and hybrid white-light properties. In addition, the dispersion and photostability of QDs in the CDNA matrix were analyzed. The colour rendering index (CRI) values and colour gamut of the QDs embedded in CDNA thin films are studied for evaluating the light quality. The results show that the ligands on the QDs enhance PLQY up to 95 and $25 \%$ in liquid and solid phases, respectively. The optical properties of the QDs in the CDNA thin films are not significantly affected by phase changes, which implies the effective hosting of QDs within CDNA. The CRI values of the mixed and stacked configuration-dependent QDs embedded in CDNA thin films are 21 and $80 \%$, respectively, which suggest the relatively stronger self-absorption of R QDs in the mixed configuration than in the stacked configuration. In addition, CRI values and colour gamut are affected by different R, G, and B QD concentrations in CDNA. These findings are important for solid-state lighting, information display systems, flexible displays, and wearable displays.

Received 28th September 2018 Accepted 8th October 2018

DOI: $10.1039 / c 8 n a 00252 e$

rsc.li/nanoscale-advances profile. ${ }^{1,2}$ The detailed photophysical properties of QDs have been reported as well. ${ }^{3}$ Colloidal QDs have applications in artificial lighting, QD LEDs, fluorescence-based microscopy, QD spectrometers, flexible lighting and displays, food science and biology, and other optoelectronic fields. ${ }^{4-9}$ At present, white-light generation using LEDs and phosphors has been widely reported, and colour quality is achieved to some extent. ${ }^{\mathbf{1 0 - 1 2}}$ However, achieving high colour purity and wide gamut practically is challenging.

Conventionally, two-dimensional synthetic polymer-based thin films with organic red, green, and blue (RGB) dyes are used for white-light generation. However, organic emitters cannot preserve their optical properties in synthetic polymers due to low stability and agglomeration. ${ }^{13}$ Furthermore, an organic emitter requires multiple photo-excitation sources for light emission due to its narrow excitation bandwidth or Stokes shift. Consequently, the use of multiple photo-excitation sources and the range of different organic emitters for different colours complicate the processing and stability of white-lightemitting devices over time. ${ }^{\mathbf{1 4 1 5}}$
${ }^{a}$ Sungkyunkwan Advanced Institute of Nanotechnology (SAINT), Sungkyunkwan University, Suwon 16419, Korea. E-mail: hchae@skku.edu; hokchung@skku.edu; sunghapark@skku.edu

${ }^{b}$ School of Chemical Engineering, Sungkyunkwan University, Suwon 16419, Korea ${ }^{c}$ Department of Physics, Sungkyunkwan University, Suwon 16419, Korea 
The major goal of this study is to construct a simple hybrid white-light-emitting optoelectronic device structure containing a single excitation source with efficient RGB emission embedded on a stable optical platform. The combination of RG with a blue LED source as well as the combination of RGB emitters with an ultraviolet (UV) LED source can significantly simplify the device system. Different combinations and arrangements of the R, G, and $\mathrm{B}$ emitters in the thin films also significantly affect the white-light spectral quality. ${ }^{\mathbf{1 6}}$

QDs have the potential to replace phosphors and can provide vivid colours and sharp contrast for future information display technology. ${ }^{17-19}$ A previous computational study has reported that QD-based lighting is more efficient than phosphor-based lighting. ${ }^{20}$ Moreover, because of the large Stokes shift range of QDs, it is possible to replace multiple excitation sources with a single excitation source in QD-based devices, which leads to simple and efficient hybrid (combination of electric and photonic) white-light emission. ${ }^{21}$

The luminescence properties of QDs are good in the liquid phase due to their colloidal stability. However, a suitable embedding matrix or platform is desirable to preserve the optical properties of QDs in the solid phase. Deoxyribonucleic acid (DNA) molecules, which have intrinsic optical characteristics (e.g., wide optical bandgap) and molecular recognition, provide a rigid platform for hosting various functional nanomaterials. ${ }^{22-25}$ Owing to the high aspect ratio and helical structure of DNA, it can provide more room for embedding luminophores with less agglomeration. ${ }^{26}$ In addition, DNA is advantageous for bio-optoelectronic applications with QD luminophores because of its biocompatible nature. ${ }^{27}$

Chemical composition-tunable gradient core/alloy/shell structured R, G, and B QDs with ligands (trioctylphosphine (TOP), tributylphosphine (TBP), and 1-dodecanethiol (DDT)) are prepared by a hot-injection single-pot synthesis method. In addition, DNA with cetyltrimethylammonium chloride (CTMA), represented as CDNA, is used for the fabrication of QDs embedded in CDNA thin films with two distinct QD configurations: mixed and orthogonally stacked QDs in CDNA. In addition, we measure the Fourier transform infrared (FTIR), PLQY, UV-vis absorbance, and photoluminescence (PL) spectra of the QDs embedded in CDNA thin films to study the ligand attachment, efficiency, excitation, and emission of the QDs, respectively. The dispersion and photostability of QDs in the CDNA matrix were analyzed.

Also, electroluminescence (EL) with different concentrations and configurations of QDs in CDNA are investigated to understand the characteristics of the white light generated by blue LEDs with RG QD films and UV LEDs with RGB QD films. Finally, the colour rendering index (CRI) and International Commission on Illumination (CIE) coordinates of the QDS embedded in CDNA thin films are discussed.

\section{Experimental}

\subsection{Preparation of TOP, TBP, and DDT ligands attached to QDs}

Luminescent R, G, and B QDs were prepared by a one-pot hotinjection method (Fig. 1(a) and Table 1). ${ }^{26}$ Cadmium oxide
(CdO), zinc acetate $\left(\mathrm{Zn}(\mathrm{Ac})_{2}\right)$, selenium (Se), sulfur (S), TOP, TBP, DDT, OA, and ODE (Sigma-Aldrich, St. Louis, USA) were used for QD synthesis. The entire process was conducted under nitrogen gas to avoid any oxidation. $\mathrm{CdO}$ and $\mathrm{Zn}(\mathrm{Ac})_{2}$ (molar quantities in Table 1) were loaded into a three-necked round bottom flask with $5 \mathrm{~mL}$ of $\mathrm{OA}$ and heated to $150{ }^{\circ} \mathrm{C}$ while stirring. We prepared two different stock solutions: Se and $\mathrm{S}$ dissolved in $3 \mathrm{~mL}$ TOP for R and G QDs (for relatively larger and medium-sized cores) and $\mathrm{S}$ dissolved in $5 \mathrm{~mL}$ ODE for B QDs (for smaller cores). At $150{ }^{\circ} \mathrm{C}, 15 \mathrm{~mL}$ ODE was added and heated further to $300{ }^{\circ} \mathrm{C}$, and the stock solution was injected rapidly. After $8 \mathrm{~min}$ of stock solution injection, $3.6 \mathrm{mM} \mathrm{S}$ dissolved in $2 \mathrm{~mL}$ TBP was slowly injected into the solution for the B QDs. Consequently, TOP (for R and G) and TBP (for B) ligands were attached on the surface of the QDs (named as $\mathrm{QD}_{\mathrm{T}}$ ). In addition, $0.5 \mathrm{~mL}$ DDT ligand was slowly injected at $290^{\circ} \mathrm{C}$ after $15 \mathrm{~min}$ of stock solution injection, forming TOP + DDT or TBP + DDT ligands on the $\mathrm{QD}$ surface $\left(\mathrm{QD}_{\mathrm{TD}}\right)$. Finally, chemical composition-tunable R, G, and B QDs with distinct ligands were obtained, $\mathrm{QD}_{\mathrm{T}}(\mathrm{R}), \mathrm{QD}_{\mathrm{TD}}(\mathrm{R}), \mathrm{QD}_{\mathrm{T}}(\mathrm{G}), \mathrm{QD}_{\mathrm{TD}}(\mathrm{G}), \mathrm{QD}_{\mathrm{T}}(\mathrm{B})$, and $\mathrm{QD}_{\mathrm{TD}}(\mathrm{B})$, which were purified and dispersed in $50 \mathrm{mg} \mathrm{mL}^{-1}$ benzene.

\subsection{Preparation of DNA and CDNA}

In $10 \mathrm{~mL}$ deionized (DI) water, $0.05 \mathrm{~g}$ DNA obtained from salmon (molecular weight of $\sim 8000 \mathrm{kDa}$, GEM Corporation, Shiga, Japan) was added. A homogeneous mixture of $0.5 \mathrm{wt} \%$ DNA solution was achieved by stirring this solution overnight at $1000 \mathrm{rpm}$ for $6 \mathrm{~h}$.

\subsection{Preparation of CDNA}

The CDNA complex was prepared according to a previously reported process with slight modifications. ${ }^{28}$ Equal concentration (0.4 wt\%) solutions of CTMA surfactant and DNA were prepared by dissolving them in DI water separately. Then CTMA was added dropwise into the DNA solution while stirring. This process allowed the attachment of the CTMA surfactant to the surface of the DNA duplexes via ionic interaction, forming a white precipitate. The precipitate was stirred for $3 \mathrm{~h}$, and then filtered and washed with excess DI water to remove unbound CTMA. Finally, the CDNA complex was obtained by washing the precipitates and drying them overnight. The CDNA solution was obtained by adding $0.3 \mathrm{~g}$ of the CDNA complex in a $10 \mathrm{~mL}$ benzene and butanol $(60: 40)$ mixture to disperse the QDs and CDNA. The final concentration of CDNA dissolved in the organic solvent was $3.0 \mathrm{wt} \%$ (Fig. 1(c)).

\subsection{Fabrication of R, G, and B QDs embedded in CDNA thin films}

$\mathrm{R}, \mathrm{G}$, and $\mathrm{B}$ QD solutions $\left(50 \mathrm{mg} \mathrm{mL}^{-1}\right.$ ) were dropped into a CDNA solution (3.0 wt\%) in ratios of 1 : 20 (for R1, G1 and B1, reference concentration) and $2: 20$ (for R2, G2 and B2, double concentration). For homogeneous mixing, the solution was vortexed and left for $1 \mathrm{~h}$. The QDs embedded in CDNA solutions were drop-cast (with $15 \mu \mathrm{L}$ drop volume) on a given substrate (glass for PL, fused silica for FTIR, PLQY, and UV-vis, and blue 


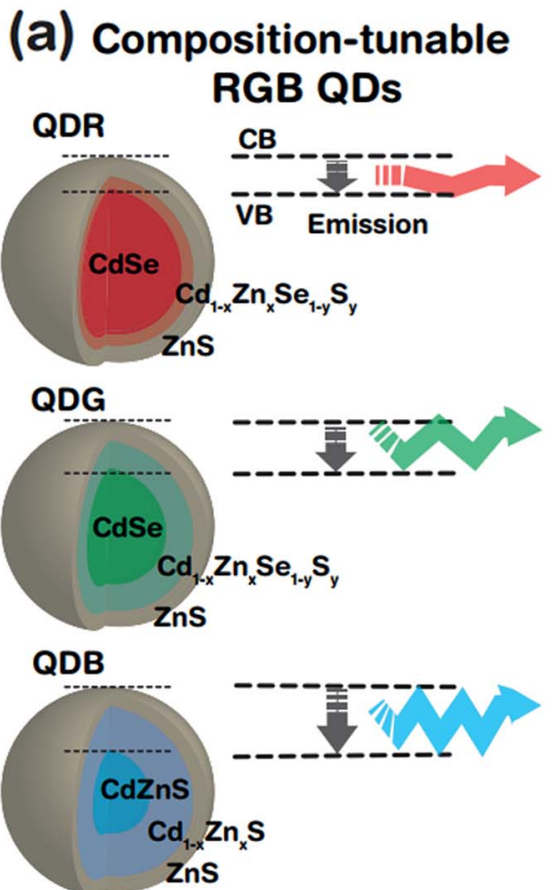

(b) Stokes Shift

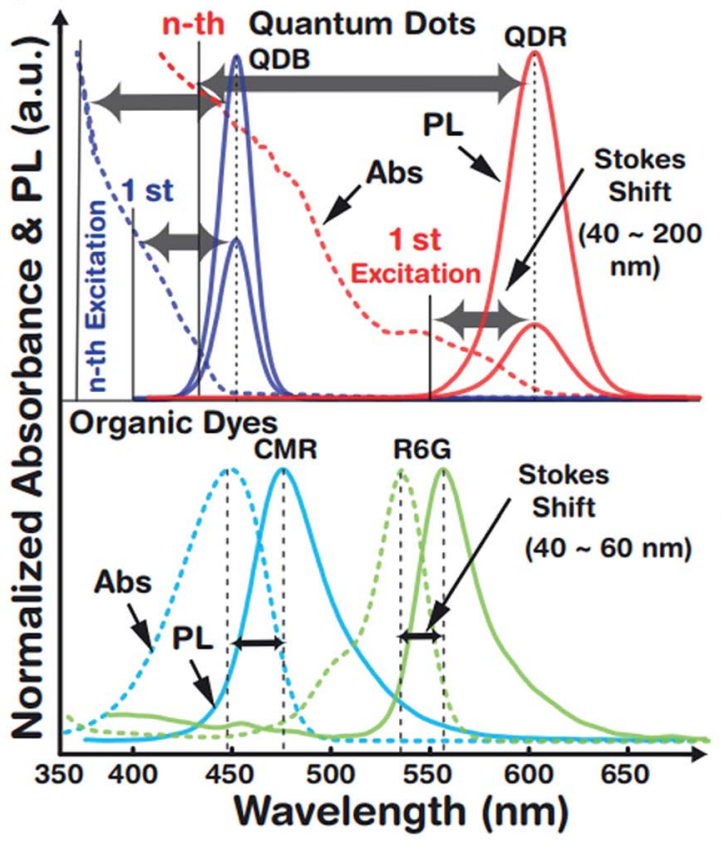

(c) DNA Structures

Pristine DNA (DNA)

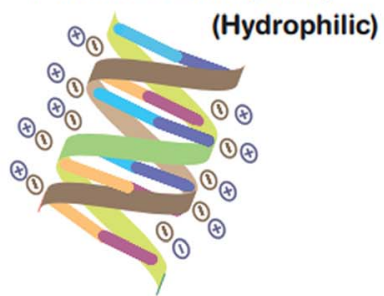

\section{CTMA-modified DNA} (CDNA)

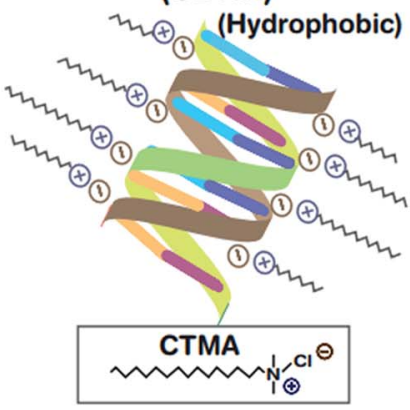

(e) Hybrid White Light

(d) QD Mixed \& Layered Thin Films
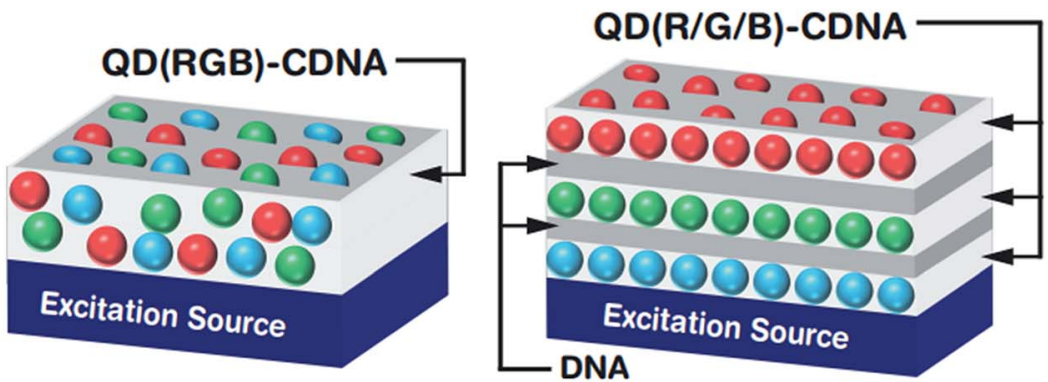

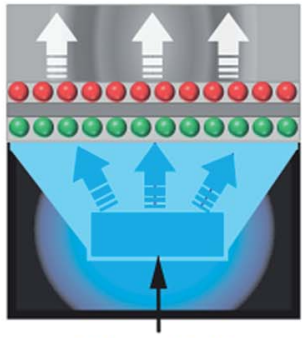

Blue LEDs

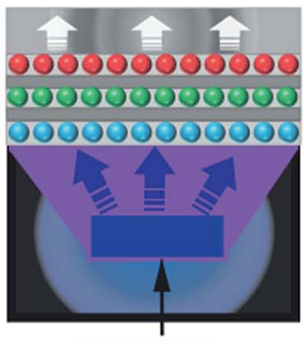

UV LEDs

Fig. 1 Schematics of the photophysical process of chemical composition-tunable QDs, comparison of Stokes shifts of QDs and organic dyes, pristine DNA and CTMA-modified DNA (CDNA) structures, fabrication of QDs embedded in CDNA thin films and excitation by blue and UV LEDs. (a) Graphical representation of composition-tunable photonic emissions emerging from QDs of different core sizes: the larger the core size, the longer the wavelength. (b) Comparison of Stokes shifts of QDs (QDBs and QDRs) and organic dyes (CMR and R6G). The QDBs and QDRs have two PL bands from the $1^{\text {st }}$ (weak) and $n^{\text {th }}$ (strong) excitation bands and wider Stokes shifts. These bands are not applicable for dyes. (c) Structures of DNA and CDNA. (d) Schematics of QDs embedded in CDNA thin films fabricated from mixed RGB QDs (QD(RGB)-CDNA) and stacked RGB QDs (QD(R/G/B)-CDNA) formed by drop casting. (e) Schematic of hybrid white-light generation using QDs embedded in CDNA thin films excited by electrically driven blue and UV LEDs.

and UV LEDs for EL). QDs embedded in CDNA thin films fabricated from mixed RGB QDs (QD(RGB)-CDNA) and stacked RGB QDs (QD(R/G/B)-CDNA) were constructed by drop casting. In addition, QDs embedded in CDNA thin films were fabricated by varying the concentration ratio of $\mathrm{R}, \mathrm{G}$, and $\mathrm{B}$ QDs. Thin layers of $0.5 \mathrm{wt} \%$ DNA (marked as /) were placed in $\mathrm{QD}(\mathrm{R} / \mathrm{G} / \mathrm{B})-$ CDNA formed by spin coating (7000 rpm) to prevent the dissolution of the bottom layers (Fig. 1(d) and Table 2).

Table 1 Chemical composition-tunable synthesis and ligand-dependent optical properties of QDs

\begin{tabular}{|c|c|c|c|c|c|c|c|}
\hline QD RGB colours & $\mathrm{CdO}(\mathrm{mM})$ & $\mathrm{Zn}(\mathrm{Ac})_{2}(\mathrm{mM})$ & $\mathrm{Se}(\mathrm{S})(\mathrm{mM})$ & $\mathrm{DDT}(\mathrm{mL})$ & Abs. $\lambda_{\max }(\mathrm{nm})$ & $\mathrm{PL} \lambda_{\max }(\mathrm{nm})$ & FWHM (nm) \\
\hline $\mathrm{QD}_{\mathrm{T}}(\mathrm{R})$ & 1.6 & 4 & $0.4(1)$ & 0 & 590 & 607 & 35 \\
\hline $\mathrm{QD}_{\mathrm{TD}}(\mathrm{R})$ & 1.6 & 4 & $0.4(1)$ & 0.5 & 590 & 608 & 35 \\
\hline $\mathrm{QD}_{\mathrm{T}}(\mathrm{G})$ & 0.3 & 4 & $0.2(3.4)$ & 0 & 510 & 531 & 45 \\
\hline $\mathrm{QD}_{\mathrm{TD}}(\mathrm{G})$ & 0.3 & 4 & $0.2(3.4)$ & 0.5 & 510 & 529 & 45 \\
\hline $\mathrm{QD}_{\mathrm{T}}(\mathrm{B})$ & 0.5 & 5 & $0(0.9)$ & 0 & 440 & 454 & 25 \\
\hline $\mathrm{QD}_{\mathrm{TD}}(\mathrm{B})$ & 0.5 & 5 & $0(0.9)$ & 0.5 & 440 & 455 & 25 \\
\hline
\end{tabular}


Table 2 R and G and R, G, and B QD ratio- and configuration-dependent EL characteristics of QDTD-CDNA thin films integrated on blue and UV LEDs

Blue LEDs $\left(\lambda_{\mathrm{EL} \max } 460 \mathrm{~nm}\right)+\mathrm{R}$ and G QD-CDNA

\begin{tabular}{lcc}
\hline $\mathrm{R}$ to $\mathrm{G}$ ratio & $\mathrm{CIE}(x, y)$ & $\mathrm{CRI}$ \\
\hline Mixed QDs embedded in single layered CDNA configuration & $(0.46,0.24)$ & 4 \\
$\mathrm{QD}_{\mathrm{TD}}(\mathrm{R} 1 \mathrm{G} 1)-\mathrm{CDNA}$ & $(0.59,0.30)$ & 30 \\
$\mathrm{QD}_{\mathrm{TD}}(\mathrm{R} 2 \mathrm{G} 1)-\mathrm{CDNA}$ & $(0.49,0.26)$ & 21
\end{tabular}

Orthogonally stacked QDs embedded in CDNA configuration

$\begin{array}{lll}\mathrm{QD}_{\mathrm{TD}}(\mathrm{R} 1 / \mathrm{G} 1)-\mathrm{CDNA} & (0.56,0.34) & 64 \\ \mathrm{QD}_{\mathrm{TD}}(\mathrm{R} 2 / \mathrm{G} 1)-\mathrm{CDNA} & (0.65,0.34) & 64 \\ \mathrm{QD}_{\mathrm{TD}}(\mathrm{R} 1 / \mathrm{G} 2)-\mathrm{CDNA} & (0.53,0.39) & 80\end{array}$
1
80 $\underline{\text { UV LEDs }\left(\lambda_{\mathrm{EL} \max } 365 \mathrm{~nm}\right)+\mathrm{R}, \mathrm{G} \text {, and B QD-CDNA }}$

R to $\mathrm{G}$ to B ratio $\quad$ CIE $(x, y) \quad$ CRI \%

$\mathrm{QD}_{\mathrm{TD}}(\mathrm{R} 1 \mathrm{G} 1 \mathrm{~B} 1)-\mathrm{CDNA}$
$\mathrm{QD}_{\mathrm{TD}}(\mathrm{R} 2 \mathrm{G} 1 \mathrm{~B} 1)-\mathrm{CDNA}$
$\mathrm{QD}_{\mathrm{TD}}(\mathrm{R} 1 \mathrm{G} 2 \mathrm{~B} 1)-\mathrm{CDNA}$
$\mathrm{QD}_{\mathrm{TD}}(\mathrm{R} 1 \mathrm{G} 1 \mathrm{~B} 2)-\mathrm{CDNA}$
$\mathrm{QD}_{\mathrm{TD}}(\mathrm{R} 2 \mathrm{G} 2 \mathrm{~B} 1)-\mathrm{CDNA}$
$\mathrm{QD}_{\mathrm{TD}}(\mathrm{R} 1 \mathrm{G} 2 \mathrm{~B} 2)-\mathrm{CDNA}$
$\mathrm{QD}_{\mathrm{TD}}(\mathrm{R} 2 \mathrm{G} 1 \mathrm{~B} 2)-\mathrm{CDNA}$

$\mathrm{QD}_{\mathrm{TD}}(\mathrm{R} 1 / \mathrm{G} 1 / \mathrm{B} 1)-\mathrm{CDNA}$

$\mathrm{QD}_{\mathrm{TD}}(\mathrm{R} 2 / \mathrm{G} 1 / \mathrm{B} 1)-\mathrm{CDNA}$

$\mathrm{QD}_{\mathrm{TD}}(\mathrm{R} 1 / \mathrm{G} 2 / \mathrm{B} 1)-\mathrm{CDNA}$

$\mathrm{QD}_{\mathrm{TD}}(\mathrm{R} 1 / \mathrm{G} 1 / \mathrm{B} 2)-\mathrm{CDNA}$

$\mathrm{QD}_{\mathrm{TD}}(\mathrm{R} 2 / \mathrm{G} 2 / \mathrm{B} 1)-\mathrm{CDNA}$

$\mathrm{QD}_{\mathrm{TD}}(\mathrm{R} 1 / \mathrm{G} 2 / \mathrm{B} 2)-\mathrm{CDNA}$

$\mathrm{QD}_{\mathrm{TD}}(\mathrm{R} 2 / \mathrm{G} 1 / \mathrm{B} 2)-\mathrm{CDNA}$

$\begin{array}{ll}(0.63,0.34) & 71 \\ (0.66,0.33) & 61 \\ (0.62,0.36) & 79 \\ (0.62,0.34) & 70 \\ (0.66,0.34) & 64 \\ (0.59,0.37) & 79 \\ (0.66,0.33) & 58\end{array}$

$(0.54,0.40)$

$(0.62,0.37)$

$(0.51,0.46)$

$(0.55,0.37)$

$(0.58,0.41)$

$(0.49,0.42)$

$(0.62,0.38)$
8

\subsection{Measurement of FTIR and PLQY spectra}

The light-matter interaction was measured by FTIR spectroscopy with an MIR_ATR detector (ZnSe) (TENSOR 27, Bruker Inc., MA, USA) in the range $3600-600 \mathrm{~cm}^{-1}$ for the QDs in the liquid phase and CDNA thin films. The QD-CDNA thin films were fabricated on a fused silica substrate to minimize the absorbance by the substrate at a relatively higher energy. The scans were averaged (32 scans) with a resolution of $4 \mathrm{~cm}^{-1}$ (Fig. 2(c)). The PLQY of the QDs in the liquid phase and CDNA thin films was measured using an integrating sphere (C9920-20, Hamamatsu Photonics, Shizuoka, Japan) via the absolute method. The PL spectra were recorded in the wavelength range $200-950 \mathrm{~nm}$ at a fixed photoexcitation wavelength of $365 \mathrm{~nm}$ (Fig. 2(d)).

\subsection{Measurement of UV-vis absorbance and PL}

The absorbance of QDs in the liquid phase and CDNA thin films fabricated on fused silica was measured using a UV-vis spectrophotometer (Cary 5G, Varian, CA, USA) by passing frequencydependent light through the QDs (Fig. 3(a)). Also, the PL spectra of the QDs in the liquid phase and CDNA thin films fabricated on glass substrates were obtained at a fixed photoexcitation wavelength of $365 \mathrm{~nm}$. The PL excitation and emission spectra were measured at room temperature using a $25 \mathrm{~W}$ Xe arc lamp equipped with a fluorometer (FS-2, Scinco, Seoul, Korea) (Fig. 3(b)).

\subsection{HRTEM, FESEM and photostability measurements}

QDRs dispersed in hexane $\left(5 \mathrm{mg} \mathrm{mL}^{-1}\right)$ and QDRs in CDNA (3.0 and $6.0 \mathrm{wt} \%$ ) solution were drop-cast on a copper mesh grid of a high-resolution transmission electron microscope (HRTEM), followed by drying for $1 \mathrm{~h}$ at $60{ }^{\circ} \mathrm{C}$. The grids with QDRs and QDRs embedded in CDNA were placed inside the HRTEM (Cs-corrected/EDS/EELS, JEM ARM 200F, JEOL Corp., Tokyo, Japan) under an ultra-vacuum for imaging. The surface degradation of QDRs embedded in CDNA thin films was measured using a field emission scanning electron microscope (FE-SEM) (JSM 7401F, JEOL Corp., Tokyo, Japan). Before the measurement, the QDR-CDNA thin film surface was coated with a platinum conductive layer $(\sim 10 \mathrm{~nm})$ by sputtering to reduce thermal damage and improve the secondary electron signals.

The photostability of QDRs embedded in CDNA was measured by exposing them to UV light (VL-6.LC, Vilber Lourmat, Suebia, Germany) having a wavelength of $254 \mathrm{~nm}(6 \mathrm{~W}$, $610 \mu \mathrm{W} \mathrm{cm}{ }^{-2}$ ) at room temperature. The sample was placed $5 \mathrm{~cm}$ away from the surface of the lamp to avoid direct thermal degradation. The setup was isolated from ambient light by placing it in a black enclosure (Fig. 4).

\subsection{Measurement and analysis of EL and white-light spectra}

EL characteristics were measured in the wavelength range 360$700 \mathrm{~nm}$ by illuminating blue or UV LEDs without and with the QD-CDNA thin films. The blue or UV LEDs were driven by a current source of $5 \mathrm{~mA}$, which was controlled by a sourcemeter (Keithley Instruments Inc., OH, USA). The LEDs with the QDCDNA thin films were mounted on an array spectrometer (MCPD-9800, Otsuka Electronics, Japan). EL data (radiation intensity, $\mathrm{W} \mathrm{sr}{ }^{-1}$ ) were collected through a half-integrating sphere. Finally, CIE colour coordinates and CRI values were calculated from the EL spectral data using the ColorCalculator simulation software (Osram Sylvania, MA, USA) (Fig. 5-7 and Table 2).

\section{Results and discussion}

\subsection{Emission wavelength-tunable QDs and Stokes shift}

The optical properties of QDs (which are treated as artificial atoms) can be easily tuned by controlling their structural parameters such as core size, chemical composition, shell 
(a) QD with TOP Ligands (QD)

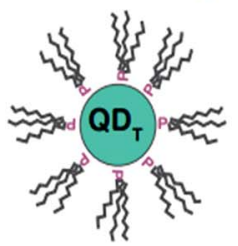

(c)

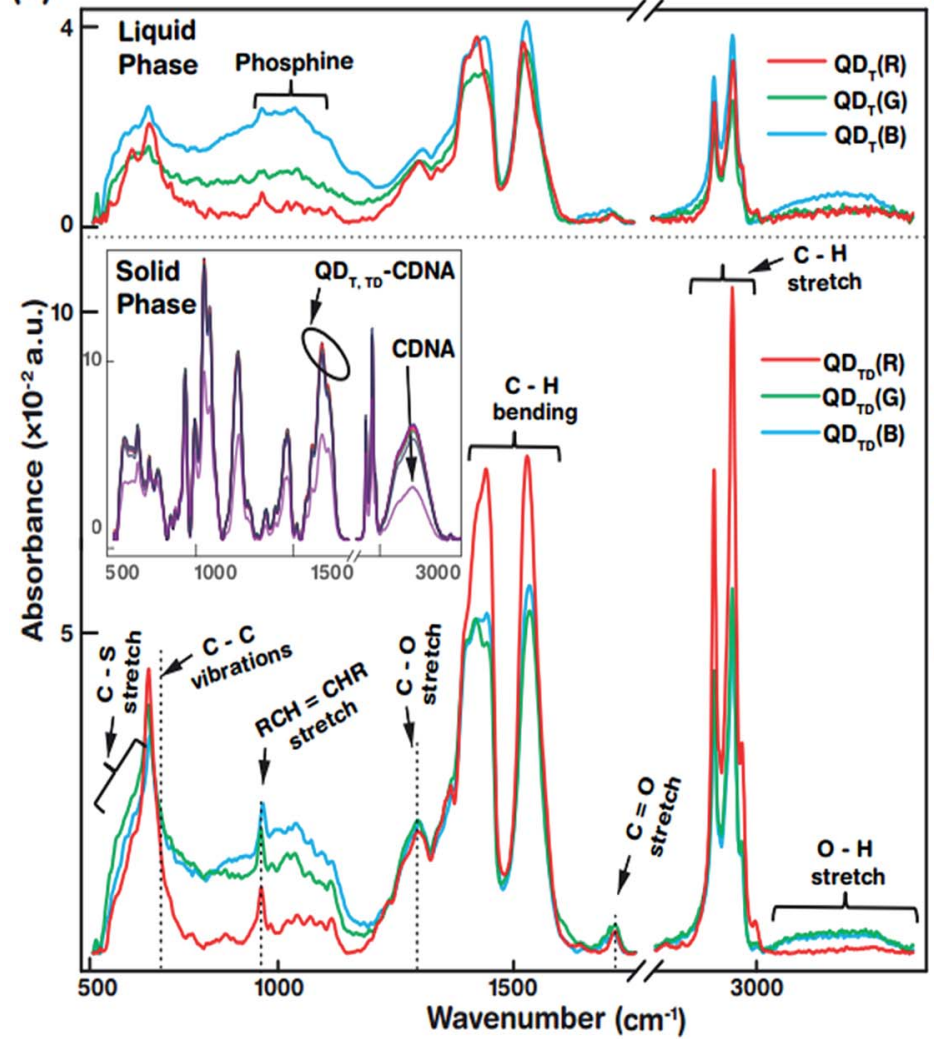

(b) QD with TOP + DDT Ligands $\left(Q_{T O}\right)$

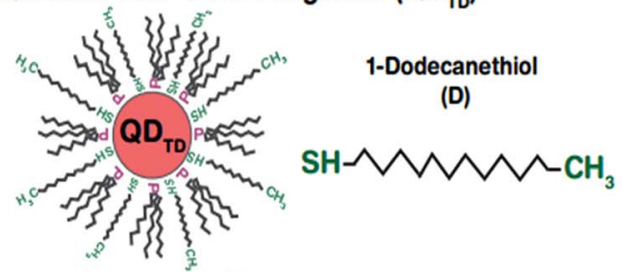

(d)

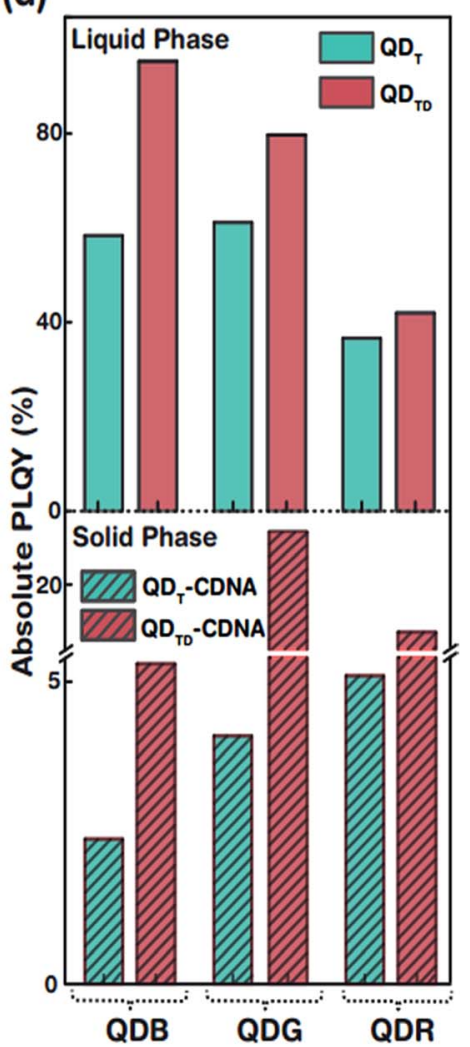

Fig. 2 Representations of T and TD ligands on QD surfaces and FTIR and PLQY bar graphs of QDs (R, G, and B) in the liquid phase and in CDNA thin films. (a and b) Schematic views of T and TD ligands attached to QDs (represented as $Q_{\text {T }}$ and $Q D_{T D}$, respectively). (c) FTIR absorbance spectra of $Q D_{T}$ (top) and $Q D_{T D}$ (bottom) in the liquid phase and of $Q D_{T}$ and $Q D_{T D}$ embedded in $C D N A$ thin films $\left(Q D_{T}-C D N A\right.$ and $Q D_{T D}-C D N A$, respectively) in the solid phase (inset). Functional groups, fingerprints of the different ligands, and their corresponding absorbance peaks are shown. The FTIR absorbance of CDNA is not noticeably altered by the QDs (inset). (d) PLQY of QDT and QD $Q_{D_{T}}-C D N A$ and $Q D_{T D}-C D N A$ in the solid phase. The PLQY values of $Q_{T D}$ are relatively higher than those of $Q D_{T}$ in both phases.

thickness, and surface ligands. The crystalline interface between the core and the shell is important to reduce the lattice mismatch and enhance the stability of the QD system. Although a well-defined core-shell QD structure constructed by a multipot synthesis process has been widely studied, this method produces a relatively large lattice mismatch and is time consuming, leading to an unstable QD system. Hence, we adopted a single-pot, hot-injection method, which allows tunable chemical composition, gradient core-shell structures, less lattice mismatch, and controllable core size. This provided a wide emission wavelength from blue to red (due to the chemical composition and core size) and a relatively stable QD system (due to the gradient core-shell interface). A schematic representation of the tunable emission properties derived from the different core sizes (related to the conduction band (CB) and valence band (VB)) and chemical compositions (e.g., CdZn) of QDs is shown in Fig. 1(a). A larger core led to a longer emission wavelength, i.e., red, green and blue photon emissions. The core size was controlled by tuning the ratio of the cadmium and selenium precursors (see the Experimental section and Table 1); increased amounts of cadmium in the presence of selenium yielded a relatively larger QD core rapidly.

Fig. 1(b) shows the absorbance spectra of QDs (red and blue QDs, expressed as QDRs and QDBs) and organic dyes (coumarin (CMR) and rhodamine 6G (R6G)), which can be used to determine the difference in Stokes shifts of the QDs and dyes. The magnitude of the Stokes shift (difference between the emission (solid line) and absorption (dotted line) maximum peak wavelengths) was relatively larger for the QDs than for the organic dyes due to dissimilar absorbance characteristics. The QDBs 
(a) Absorbance

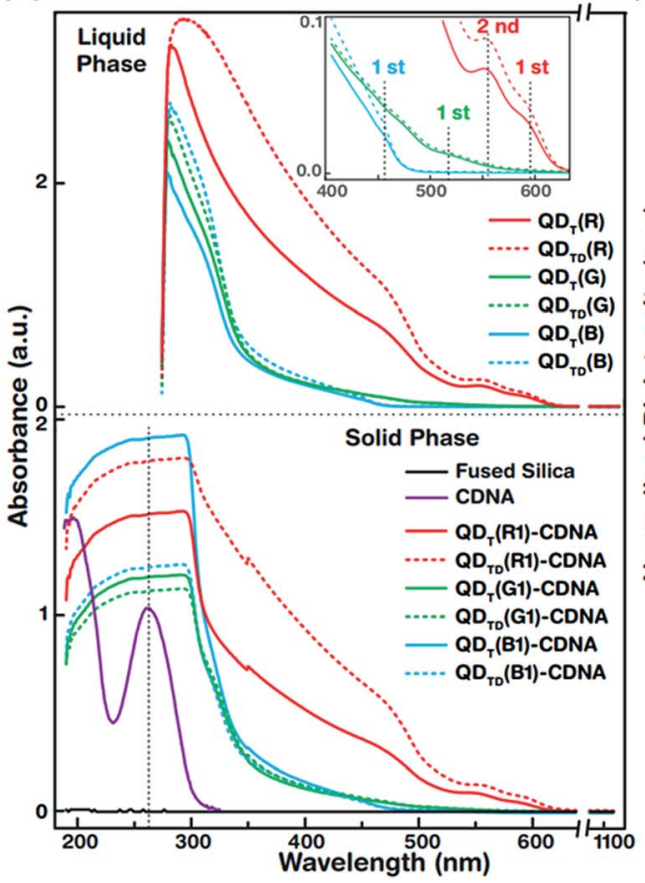

(b) Photoluminescence (PL)

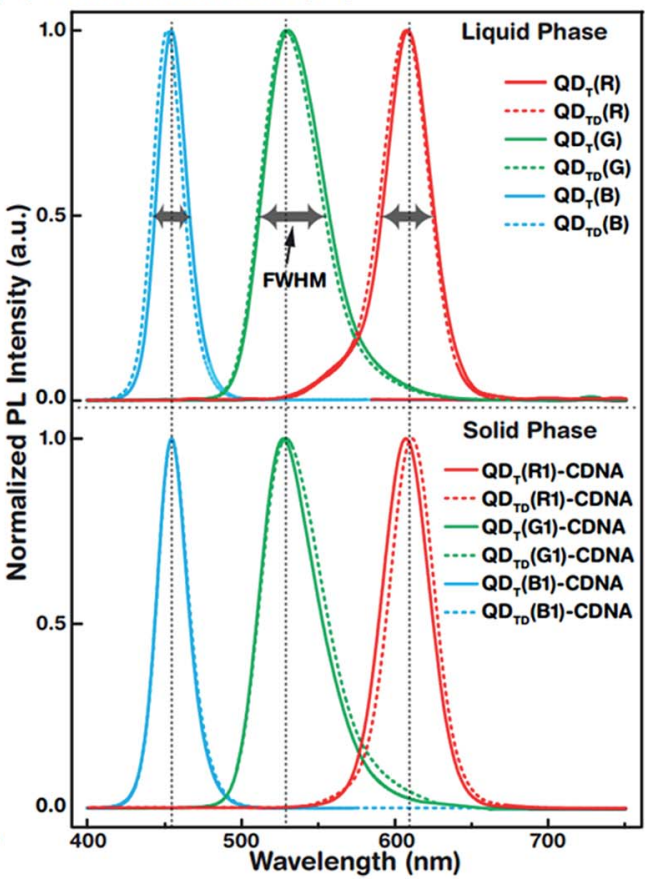

Fig. 3 Optical spectra of QDs in the liquid phase and of QDs embedded in DNA thin films. UV-vis absorbance and PL spectra of R, G, and B QDs with either T or TD ligands $\left(Q_{T}\right.$ or $Q_{T D}$, respectively) and QDs embedded in DNA thin films with either T or TD ligands (QD,-CDNA or $\mathrm{QD}_{\mathrm{TD}}$-CDNA, respectively) are shown by solid and dotted lines, respectively (details in the Experimental section). (a) UV-vis absorbance spectra of $Q_{T}$ and $Q D_{T D}$ and $Q D_{T}-C D N A$ and $Q D_{T D}-C D N A$. The characteristic DNA absorbance peak at around $260 \mathrm{~nm}$ is shown by a vertical dotted line (bottom) (inset). The first absorbance maximum peaks of $Q D_{T, T D}(R), Q D_{T, T D}(G)$, and $Q D_{T, T D}(B)$ are observed at wavelengths of 590,510 , and $440 \mathrm{~nm}$, respectively. Interestingly, the second absorbance peak of $Q_{D T, T D}(R)$ is clearly visible at $560 \mathrm{~nm}$. The QD absorbance peak positions in QDT-CDNA and QD TD-CDNA are similar to those in QDT and QDTD in the liquid phase. (b) Normalized PL spectra of QDT and QDTD and $Q_{D_{T}}-C D N A$ and $Q D_{T D}-C D N A . Q D_{T, T D}(G)$ has a relatively wider FWHM than the other samples. Reference concentrations of $Q D s$ for $R, G$, and $B$ in CDNA are used and marked as R1, G1, and B1, respectively.

(a) QDR without CDNA

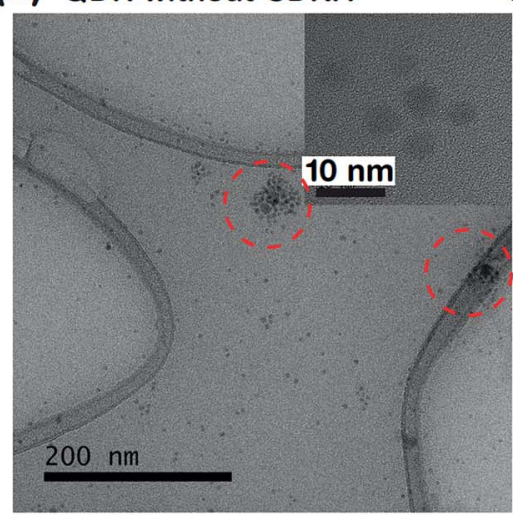

QDR with CDNA

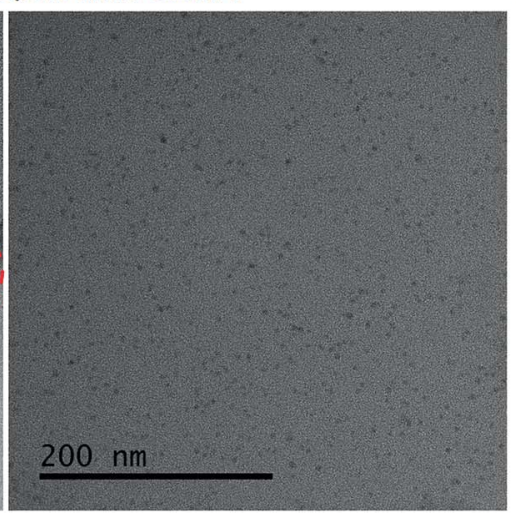

\section{(b) Before}

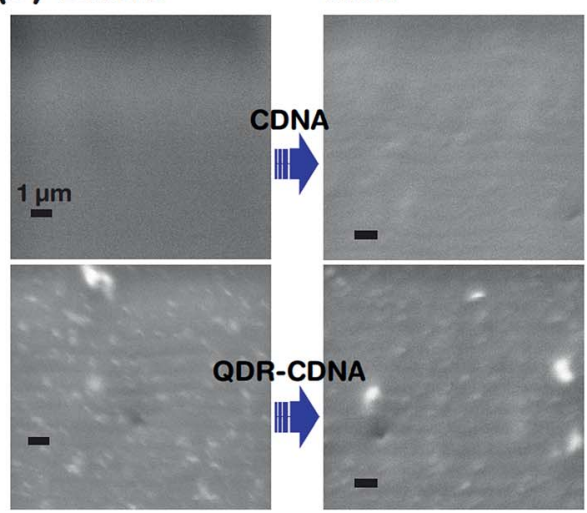

Fig. 4 Dispersion and UV light degradation analysis of QDRs embedded in CDNA thin films. (a) HRTEM images of QDRs without and with the CDNA matrix: (left) QDRs without CDNA show aggregation and (right) QDRs in CDNA show moderate dispersion. The inset in the left image indicates the particle size of QDRs $(\sim 6.7 \mathrm{~nm}$ ). (b) Surface degradation analysis of (top) CDNA and (bottom) QDRs embedded in a CDNA thin film by FESEM. The FESEM images show minute surface degradation after $20 \mathrm{~h}$ of UV light exposure.

and QDRs have two PL bands from the $1^{\text {st }}$ (weak) and $n^{\text {th }}$ (strong) excitation bands and wider Stokes shifts. The continuous absorbance characteristics of the QDs allowed luminescence excitation using a UV or green-light excitation source, ${ }^{29}$ which indicated that the absorbance spectra of QDRs ranged from 300 to $590 \mathrm{~nm}$. Consequently, QDRs and QDGs could be excited by a single blue LED and all QDs (QDRs, QDGs, and QDBs) could be excited by a UV LED. In contrast, the organic dyes rarely showed continuous absorbance properties in the high-energy region and hence had limited Stokes shifts. 


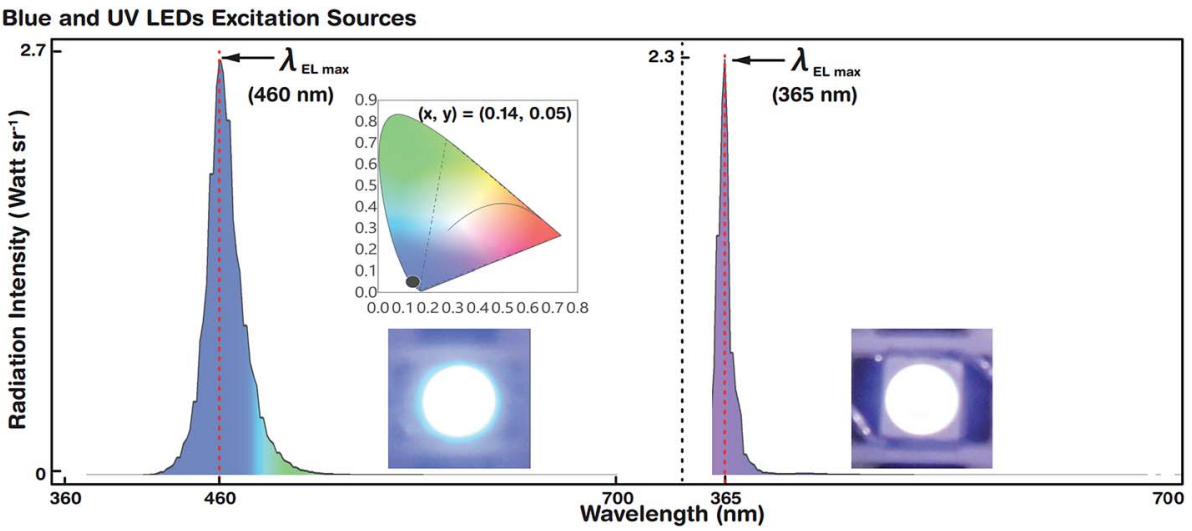

Fig. 5 EL spectra and colour coordinates of reference blue and UV LEDs. The reference blue (left) and UV (right) excitation sources and CIE colour coordinates are shown in the insets. The maximum EL peak wavelengths ( $\lambda_{E L}$ max $)$ of the blue and UV LEDs are observed at 460 and $365 \mathrm{~nm}$, respectively. FWHM values of blue and UV LEDs are $27 \mathrm{~nm}$ and $15 \mathrm{~nm}$, respectively. CIE colour coordinates of blue LEDs are (0.14, 0.05$)$.

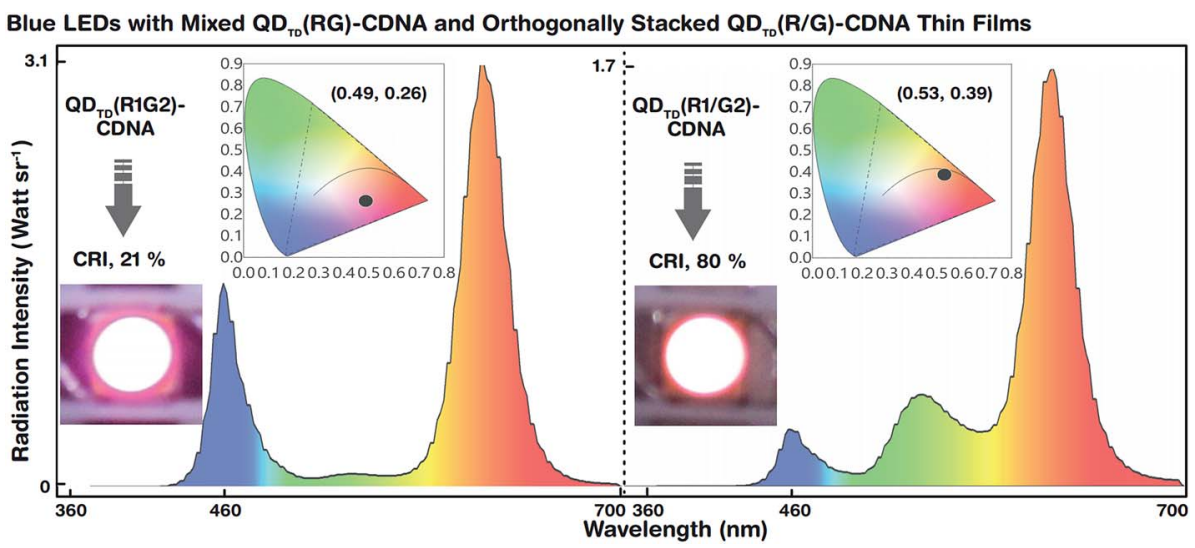

Fig. 6 EL spectra of blue LEDs with QDs embedded in CDNA thin films with mixed and orthogonally stacked configurations. The white-light luminescence spectra and CIE colour coordinates are shown in the insets. White-light radiation intensities emitted by (left) a mixture of reference concentration of R (R1) and double concentration of G (G2) QDs embedded in CDNA (QD TD (R1G2)-CDNA) and (right) stacked R1 and G2 QDs embedded in CDNA thin films $\left(Q D_{T D}(R 1 / G 2)-C D N A\right)$ integrated with a blue LED. Although the $R$ to $G$ QD concentration ratio (1:2) in QD TD $(R 1 G 2)-C D N A$ and $Q D_{T D}(R 1 / G 2)-C D N A$ is the same, their CRI values are considerably different. This indicates that the self-absorption of QDs in the $Q D_{T D}(R 1 G 2)-C D N A$ configuration is greater than that in the $\mathrm{QD}_{T D}(\mathrm{R} 1 / \mathrm{G} 2)-\mathrm{CDNA}$ configuration.

QDs embedded in CDNA thin films fabricated from mixed RGB QDs (named as QD(RGB)-CDNA) and stacked RGB QDs $(\mathrm{QD}(\mathrm{R} / \mathrm{G} / \mathrm{B})$-CDNA) are shown in Fig. 1(d). Thin layers of DNA (marked as /) formed by spin coating were sandwiched between the $\mathrm{QD}(\mathrm{R} / \mathrm{G} / \mathrm{B})-\mathrm{CDNA}$ layers to avoid the dissolution of the bottom layers (a detailed discussion can be found in our previous report). ${ }^{30}$ Fig. 1 (e) shows a schematic of the hybrid white light generated by the QDs embedded in CDNA thin films.

\subsection{Ligand-dependent PLQY characteristics of individual $R$, G, and B QDs in the liquid phase and CDNA thin films}

The specific morphological and photophysical properties of colloidal QDs strongly depend on the ligands attached to them. Ligands act as stabilizing agents (to prevent agglomeration and regulate the diffusion kinetics during synthesis) and dispersing agents, i.e., they facilitate dispersion in liquid and solid media to prevent the self-aggregation of QDs. The ligands attached to the QD surface interact with the dispersion medium or template
(CDNA thin films) via electrostatic and van der Waals forces. In particular, the dispersion of QDs with thiol ligands in a solid medium has been reported to lead to improved stability and PLQY due to the optimal dispersion of QDs..$^{31,32}$ Consequently, appropriate ligands on the QD surface can significantly enhance the photophysical characteristics of QDs in liquid and solid phases. In this study, we used oleic acid (OA) and 1-octadecene (ODE) ligands and TOP for R and G or TBP for B (both TOP and TBP are represented as T) and 1-dodecanethiol (DDT is represented as D) ligands for the synthesis and dispersion of QDs in both chloroform solution and CDNA thin films (Fig. 2(a and b)). D ligands, composed of a thiol group at the end of the chain, served as additional passivation ligands after $\mathrm{T}$ stabilized the QDs in the liquid and solid phases. ${ }^{33}$ CDNA could be used as an efficient template for QDs in solid films due to its unique polymeric nature (rigidity, transparency, and biocompatibility).

Fig. 2(c) shows the Fourier transform infrared (FTIR) absorbance spectra of $\mathrm{QD}_{\mathrm{T}}$ and $\mathrm{QD}_{\mathrm{TD}}$ in the liquid phase and of $\mathrm{QD}_{\mathrm{T}}$ 
(a) UV LEDs with Mixed $Q D_{\text {TD }}(R G B)$-CDNA Thin Films

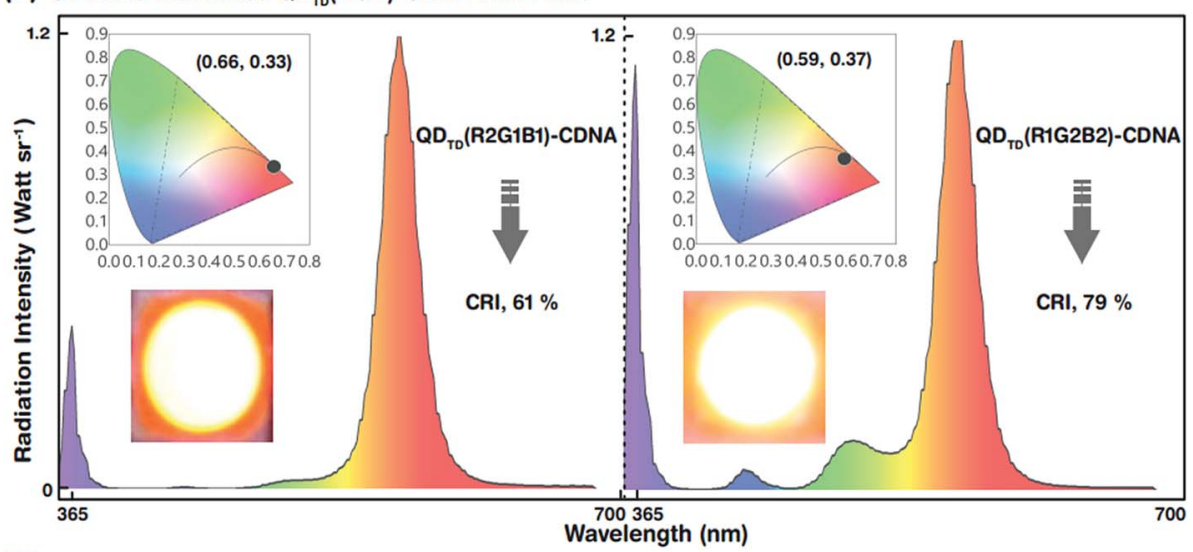

(b) UV LEDs with Orthogonally Stacked $Q D_{\text {To }}(R / G / B)$-CDNA Thin Films

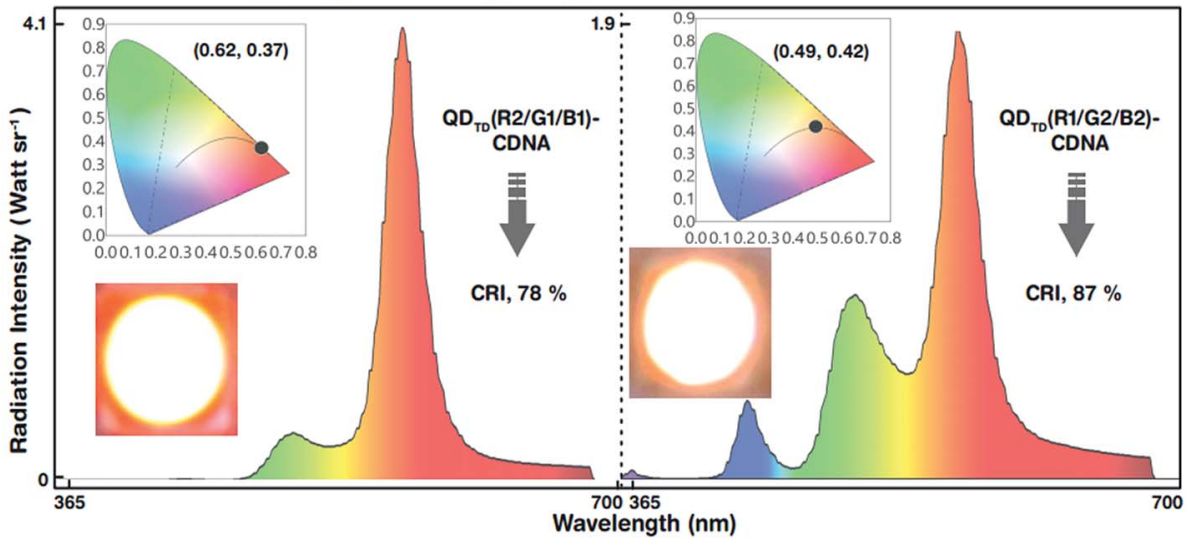

Fig. 7 White-light radiation intensities emitted by mixed R, G, and B QDs embedded in CDNA and stacked R, G, and B QDs embedded in CDNA thin films integrated with a UV LED. Two different sets of QD concentrations (R2, G1, B1 and R1, G2, B2) are used in the mixed and stacked configurations. In addition, the device white-light luminescence photographs and CIE colour coordinates are shown in the insets. (a) White-light spectra of $Q D_{T D}(R 2 G 1 B 1)-C D N A$ and $Q D_{T D}(R 1 G 2 B 2)-C D N A$ excited by the UV LED. Even though the configuration is the same, different $R$, G, and $B$ QD concentrations in $Q D_{T D}$ (R2G1B1)-CDNA and $Q D_{T D}$ (R1G2B2)-CDNA lead to an $\sim 18 \%$ CRI increase because of the significant change in selfabsorption of R QDs. (b) White-light spectra of QDTD (R2/G1/B1)-CDNA and QDTD(R1/G2/B2)-CDNA excited by the UV LED. The CRI values of $\mathrm{QD}_{\mathrm{TD}}$ (R2/G1/B1)-CDNA and $\mathrm{QD}_{\mathrm{TD}}$ (R1/G2/B2)-CDNA are 78\% and 87\%, respectively. The CRI increases because of the change in R, G, and B QD concentrations. Qualitatively, the device luminescence photographs agree well with the CIE colour coordinates.

and $\mathrm{QD}_{\mathrm{TD}}$ embedded in CDNA thin films (represented as $\mathrm{QD}_{\mathrm{T}^{-}}$ CDNA and $\mathrm{QD}_{\mathrm{TD}}-\mathrm{CDNA}$ ) in the solid phase. As expected, the FTIR absorbance peak intensities varied with functional groups and alkane chains, confirming the presence of ligands on the QDs. The functional groups originating from OA, ODE, T, and D ligands were identified as follows. The medium absorbance peaks at $630-660 \mathrm{~cm}^{-1}$ corresponded to the C-S stretching of thiol, presumably originating from D. Broad peaks corresponding to inorganic elemental phosphine were observed at 1000-1100 $\mathrm{cm}^{-1}$. The broad peaks at $700-1300 \mathrm{~cm}^{-1}$ corresponded to the $\mathrm{C}-\mathrm{C}$ skeletal vibrations of methyne. The narrow short peaks at $965-975 \mathrm{~cm}^{-1}$ were attributed to the $\mathrm{RCH}=\mathrm{CHR}$ ( $\mathrm{R}$ refers to methyl) vibrations of OA. Ligands possessing $\mathrm{C}-\mathrm{H}$ chains showed relatively strong absorbance peaks. The strong peaks at 1445-1485 $\mathrm{cm}^{-1}$ and 2848 and $2921 \mathrm{~cm}^{-1}$ corresponded to the $\mathrm{C}-\mathrm{H}$ bending of methylene and the $\mathrm{C}-\mathrm{H}$ asymmetric stretching of methyl and methylene, respectively. In contrast, the minor peaks at 1310 and $1720 \mathrm{~cm}^{-1}$ corresponded to the $\mathrm{C}-\mathrm{O}$ and $\mathrm{C}=\mathrm{O}$ stretching of $\mathrm{OA}$. Finally, the $\mathrm{OH}$ stretching of OA was observed as a broad peak at 3200$3570 \mathrm{~cm}^{-1} \cdot{ }^{34}$ The FTIR absorbance spectra of the QDS embedded in CDNA thin films in the solid phase are shown in the inset of Fig. 2(c). FTIR absorbance of the QD-CDNA thin films exhibited the characteristic behaviour of pristine CDNA. The FTIR absorbance of CDNA clearly showed characteristic peaks for sugar, phosphate groups (1250-600 $\left.\mathrm{cm}^{-1}\right)$, and nucleotide bases (1800-1300 $\mathrm{cm}^{-1}$ ); detailed mode assignments are shown in our previous report. ${ }^{24}$ As expected, D ligands formed a stronger bond on the QD surface because they had more free electrons (about 2 times) than T. The optical properties of QDs with different ligands were found to be different due to the degree of surface passivation and dispersion in liquid and solid media.

The PLQY of $\mathrm{QD}_{\mathrm{T}}$ and $\mathrm{QD}_{\mathrm{TD}}$ in the liquid phase and of $\mathrm{QD}_{\mathrm{T}^{-}}$ CDNA and $\mathrm{QD}_{\mathrm{TD}}-\mathrm{CDNA}$ in the solid phase is shown in Fig. 2(d). The PLQY values of QDRs, QDGs, and QDBs with T (TD) ligands in the liquid phase were about 37,61 , and $58 \%(42,80$, and 95\%), respectively. Similarly, the PLQY values of QDRs, QDGs, 
and QDBs with $\mathrm{T}$ (TD) ligands in the CDNA thin films were about 5, 4, and 3\% $(17,25$, and $6 \%)$, respectively. Although QDBs showed a higher PLQY than QDRs in the liquid phase, QDGs showed a higher PLQY than QDBs in the CDNA thin films. This might be due to the variation in the QD shell thickness. Interestingly, QDs with both $\mathrm{T}$ and $\mathrm{D}$ ligands $\left(\mathrm{QD}_{\mathrm{TD}}\right)$ showed an enhanced PLQY in both phases compared to QDs with only $\mathrm{T}$ ligands $\left(\mathrm{QD}_{\mathrm{T}}\right)$ because of the efficient passivation and removal of dangling bonds. ${ }^{35}$ CDNA duplexes (with a high aspect ratio) served as good passivation materials as they provided stable surroundings for QDs because of their flexible duplex networks. Consequently, the CDNA templates prevented aggregation of QDs by acting as effective spacers and increased the loading efficiency of QDs. ${ }^{12,36}$

\subsection{Optical properties of individual R, G, and B QDs in the liquid phase and CDNA thin films}

The UV-vis absorbance spectra of $\mathrm{QD}_{\mathrm{T}}$ (solid lines) and $\mathrm{QD}_{\mathrm{TD}}$ (dotted lines) in the liquid phase (top) and the CDNA thin films (bottom) are shown in Fig. 3(a). The absorbance spectra were recorded in the wavelength range 190-1100 nm. The absorbance peaks of the QDs (inset) and CDNA (around $260 \mathrm{~nm}$ ) thin films are shown by vertical dotted lines. Although the characteristic peak positions did not change, $\mathrm{QD}_{\mathrm{T}}$ and $\mathrm{QD}_{\mathrm{TD}}$ showed different absorbance intensities in the liquid phase and in CDNA thin films. As shown in the inset, the spectra of $\mathrm{QD}_{\mathrm{T}, \mathrm{TD}}(\mathrm{R}), \mathrm{QD}_{\mathrm{T}, \mathrm{TD}}(\mathrm{G})$, and $\mathrm{QD}_{\mathrm{T}, \mathrm{TD}}(\mathrm{B})$ showed the first absorbance maximum at wavelengths of around 590, 510, and $440 \mathrm{~nm}$ (second absorbance maximum of $\mathrm{QD}_{\mathrm{T}, \mathrm{TD}}(\mathrm{R})$ at $560 \mathrm{~nm}$ ), respectively. The absorbances of QDs in the liquid phase gradually increased toward shorter wavelengths up to $280 \mathrm{~nm}$. The absorbance of QD-CDNA thin films exhibited the characteristic behavior of QDs and pristine CDNA. The QD absorbance characteristic peak positions in $\mathrm{QD}_{\mathrm{T}}-\mathrm{CDNA}$ and $\mathrm{QD}_{\mathrm{TD}}-\mathrm{CDNA}$ were similar to those in $\mathrm{QD}_{\mathrm{T}}$ and $\mathrm{QD}_{\mathrm{TD}}$ in the liquid phase. Here, the reference concentrations of R, G, and B QDs in the CDNA thin films were used and marked as R1, G1, and B1, respectively (detailed in the Experimental section).

Fig. 3(b) displays the normalized PL spectra of $\mathrm{QD}_{\mathrm{T}}$ and $\mathrm{QD}_{\mathrm{TD}}$ in the liquid phase (top) and $\mathrm{QD}_{\mathrm{T}}-\mathrm{CDNA}^{-}$and $\mathrm{QD}_{\mathrm{TD}^{-}}-\mathrm{CDNA}$ thin films (bottom) measured in the range 400-750 $\mathrm{nm}$. The PL characteristic emission peaks and full width at half maximum (FWHM) values are shown by vertical dotted lines and horizontal arrows, respectively. $\mathrm{QD}_{\mathrm{T}, \mathrm{TD}}(\mathrm{G})$ in the liquid phase and in CDNA showed a relatively wider FWHM than the other samples (details in Table 1). The PL peak positions of $\mathrm{QD}_{\mathrm{T}}$ and $\mathrm{QD}_{\mathrm{TD}}$ in the liquid phase and $\mathrm{QD}_{\mathrm{T}}-\mathrm{CDNA}$ and $\mathrm{QD}_{\mathrm{TD}}-\mathrm{CDNA}$ thin films did not change significantly (less than $2 \mathrm{~nm}$ ). The $\mathrm{PL}$ emission area (proportional to PLQY) of $\mathrm{QD}_{\mathrm{T}}, \mathrm{QD}_{\mathrm{TD}}, \mathrm{QD}_{\mathrm{T}}-\mathrm{CDNA}$, and $\mathrm{QD}_{\mathrm{TD}}-\mathrm{CDNA}$ varied with individual QDs and was strongly dependent on the ligands.

\subsection{Dispersion and photostability analysis of QDRs embedded in CDNA thin films}

Fig. 4 shows the dispersion and UV light degradation analysis of QDRs embedded in CDNA thin films. The HRTEM image of
QDRs without CDNA clearly showed the aggregation of QDRs. On the other hand, QDRs with the CDNA matrix revealed a moderate and uniform distribution of QDRs in the CDNA thin film. Here, CDNA (which provided surface passivation of QDs) could effectively reduce the surface energy and kinetic motion of QDs. Consequently, CDNA prevents their aggregation. The better dispersion of QDs in CDNA obviously leads to higher PLQY, which is in good agreement with the discussion of PLQY in Section 3.2.

FESEM analysis is carried out to understand the potential surface damage and degradation of CDNA and QDs embedded in CDNA thin film surfaces under UV light exposure (i.e., harsh environment). The FESEM images indicated the stability of QDS embedded in CDNA thin films after 20 h of exposure to UV light (Fig. 4b). There is no visible damage (e.g., cracking of the surface) in the FE-SEM images for both CDNA and QDS embedded in CDNA thin films. Hence, CDNA acts as an efficient matrix (i.e., host) for QDs even under the accelerated photodegradation conditions.

\subsection{EL spectra and colour coordinates of reference blue and UV LEDS}

Blue and UV LED photoexcitation sources for down-conversion of white light were used for EL spectral characterization. Fig. 5 shows the luminescence spectra and colour coordinates of the reference blue and UV LEDs. The LEDs emitted at a maximum EL peak wavelength $\left(\lambda_{\mathrm{EL} \text { max }}\right)$ of 460 and $365 \mathrm{~nm}$ with a driving voltage (current) of 2.6 V (@5 mA) and3.2 V (@5 mA), respectively. The FWHM of blue and UV LEDs was 27 and $15 \mathrm{~nm}$, respectively.

For blue LEDs, the CIE colour coordinates were $(0.14,0.05)$. We designed the experiment such that white light was generated by R, G, and B primary colours. The blue LED as the B emitting source also served as a photoexcitation source for R and G QDs. However, the UV LED merely acted as a photoexcitation source for R, G, and B QDs to produce hybrid (combination of EL and PL) white light by the down-conversion phenomenon (via Stokes shift). The blue and UV LEDs were stable and showed Gaussianlike spectral emission distributions.

\subsection{EL spectra of QDs embedded in CDNA thin films with mixed and orthogonally stacked configurations integrated on blue LEDs}

Two configurations, mixed and orthogonally arranged R and G QD structures, were obtained with different concentrations of $\mathrm{R}$ and $\mathrm{G}$ QDs. $\mathrm{R}$ and $\mathrm{G} \mathrm{QD}_{\mathrm{TD}}$ embedded in CDNA thin films at three different concentration ratios $(1: 1,2: 1$, and $1: 2)$ of QDs were obtained either by mixing, denoted as $\mathrm{QD}_{\mathrm{TD}}(\mathrm{R} 1 \mathrm{G} 1)-$, $\mathrm{QD}_{\mathrm{TD}}(\mathrm{R} 2 \mathrm{G} 1)-$, and $\mathrm{QD}_{\mathrm{TD}}(\mathrm{R} 1 \mathrm{G} 2)-\mathrm{CDNA}$, respectively, or by orthogonally stacking, denoted as $\mathrm{QD}_{\mathrm{TD}}(\mathrm{R} 1 / \mathrm{G} 1)-, \mathrm{QD}_{\mathrm{TD}}(\mathrm{R} 2 / \mathrm{G} 1)-$, and $\mathrm{QD}_{\mathrm{TD}}(\mathrm{R} 1 / \mathrm{G} 2)-\mathrm{CDNA}$, respectively, and were integrated on blue LEDs for EL measurement in the wavelength range 360$700 \mathrm{~nm}$. Although a CDNA thin film with a mixture of R and G QDs could form a single layer, layers formed with the orthogonal stacking configuration for R and G QDs could form double layers too. However, a DNA layer without CTMA was introduced 
between the CDNA layers to avoid the dissolution of the bottom layers by the 1-butanol solvent in CDNA during stacking. Here, the hydrophilic DNA layer effectively separated the R and G $\mathrm{QD}_{\mathrm{TD}}$ embedded in CDNA layers.

The white light spectra and CIE colour coordinates of the mixed $\mathrm{QD}_{\mathrm{TD}}(\mathrm{R} 1 \mathrm{G} 2)-\mathrm{CDNA}$ and orthogonally stacked $\mathrm{QD}_{\mathrm{TD}}(\mathrm{R} 1 / \mathrm{G} 2)-\mathrm{CDNA}$ thin films integrated on blue LEDs are shown in Fig. 6. Although the concentration ratio $(1: 2)$ of $\mathrm{R}$ to $\mathrm{G}$ was the same, their CRI (CIE) values were significantly different, $21 \%(0.49,0.26)$ and $80 \%(0.53,0.39)$, respectively. This difference may originate because of suppression of the $G$ QD intensity. Although the G QD concentration was twice that of the R QDs, the spectral wavelengths of the latter were relatively dominant in $\mathrm{QD}_{\mathrm{TD}}(\mathrm{R} 1 \mathrm{G} 2)$-CDNA. This indicated the selfabsorption nature of the R QDs. Consequently, the R QDs absorbed $G$ light more in the mixed R and G QDs than in the stacked structures. The R QDs in the mixed structures showed high-energy continuous absorbance involving G spectral energy emission and inter-particle resonance absorption. ${ }^{37,38}$ In contrast, the self-absorption of R QDs in the stacked configuration was relatively smaller than that in the mixed configuration. Similar self-absorption trends were observed at the other $\mathrm{R}$ and G QD concentration ratios of $1: 1$ and $2: 1$ with mixed and stacked $\mathrm{QD}_{\mathrm{TD}}$-CDNA thin films (detailed CRI values and CIE colour coordinates are shown in Table 2). We concluded that the configuration and concentration ratio of the QDs, which could be easily controlled, also significantly affected the spectral distribution.

\subsection{EL spectra of CDNA thin films with different R, G, and $B$ QD concentrations integrated on UV LEDs}

White-light spectra of the QDs embedded in CDNA thin films with seven different $\mathrm{R}, \mathrm{G}$, and $\mathrm{B}$ QD concentration ratios $(1: 1: 1$, $2: 1: 1,1: 2: 1,1: 1: 2,2: 2: 1,1: 2: 2$, and $2: 1: 2)$ with mixed, $\quad \mathrm{QD}_{\mathrm{TD}}(\mathrm{R} 1 \mathrm{G} 1 \mathrm{~B} 1)-, \quad \mathrm{QD}_{\mathrm{TD}}(\mathrm{R} 2 \mathrm{G} 1 \mathrm{~B} 1)-\mathrm{QD}_{\mathrm{TD}}(\mathrm{R} 1 \mathrm{G} 2 \mathrm{~B} 1)-$, $\mathrm{QD}_{\mathrm{TD}}(\mathrm{R} 1 \mathrm{G} 1 \mathrm{~B} 2)-, \mathrm{QD}_{\mathrm{TD}}(\mathrm{R} 2 \mathrm{G} 2 \mathrm{~B} 1)-\mathrm{QD}_{\mathrm{TD}}(\mathrm{R} 1 \mathrm{G} 2 \mathrm{~B} 2)-$, and $\mathrm{QD}_{\mathrm{TD}}$ (R2G1B2)-CDNA, and orthogonally stacked, $\mathrm{QD}_{\mathrm{TD}}(\mathrm{R} 1 / \mathrm{G} 1 / \mathrm{B} 1)-$, $\mathrm{QD}_{\mathrm{TD}}(\mathrm{R} 2 / \mathrm{G} 1 / \mathrm{B} 1)-, \quad \mathrm{QD}_{\mathrm{TD}}(\mathrm{R} 1 / \mathrm{G} 2 / \mathrm{B} 1)-, \quad \mathrm{QD}_{\mathrm{TD}}(\mathrm{R} 1 / \mathrm{G} 1 / \mathrm{B} 2)-, \quad \mathrm{QD}_{\mathrm{TD}}$ (R2/G2/B1)-, $\quad \mathrm{QD}_{\mathrm{TD}}(\mathrm{R} 1 / \mathrm{G} 2 / \mathrm{B} 2)-$, and $\mathrm{QD}_{\mathrm{TD}}(\mathrm{R} 2 / \mathrm{G} 1 / \mathrm{B} 2)-\mathrm{CDNA}$, configurations integrated on UV LEDs were measured in the wavelength range 360-700 $\mathrm{nm}$ (Fig. 7). The white light was generated by the combination of $\mathrm{R}, \mathrm{G}$, and $\mathrm{B}$ primary colours via excitation of the R, G, and B QDs using UV LEDs. The UV LEDs functioned as excitation sources of the R, G, and B QDs and produced hybrid white light by the down-conversion process.

The white-light spectra, CRI, and CIE colour coordinates of the mixed-layered $\mathrm{QD}_{\mathrm{TD}}(\mathrm{R} 2 \mathrm{G} 1 \mathrm{~B} 1)$ - and $\mathrm{QD}_{\mathrm{TD}}(\mathrm{R} 1 \mathrm{G} 2 \mathrm{~B} 2)-\mathrm{CDNA}$ thin films excited by the UV LED are shown in Fig. 7(a). Their CRI values were 61 and $79 \%$, respectively. Although the configuration was the same, the different R, G, and B QD concentration ratios of $2: 1: 1$ and $1: 2: 2$ in CDNA led to an approximately $18 \%$ CRI increase because of the significant change in the self-absorption of R QDs. This implied that the self-absorption of the R QDs could be minimized using excess $\mathrm{G}$ and B QDs. Consequently, the CRI efficiency could be improved easily by controlling the R, G, and B QD concentrations. The EL,
CRI, and CIE colour coordinates of the triple-layered $\mathrm{QD}_{\mathrm{TD}}(\mathrm{R} 2 / \mathrm{G} 1 / \mathrm{B} 1)-$ and $\mathrm{QD}_{\mathrm{TD}}(\mathrm{R} 1 / \mathrm{G} 2 / \mathrm{B} 2)-\mathrm{CDNA}$ thin films excited by the UV LED are shown in Fig. 7(b). We observed that the CRI of the orthogonally stacked thin films was enhanced compared to that of the mixed thin films, which indicated that the R QD intensity could be controlled by curtailing the selfabsorption. The CRI values for the $\mathrm{QD}_{\mathrm{TD}}(\mathrm{R} 2 / \mathrm{G} 1 / \mathrm{B} 1)-$ and $\mathrm{QD}_{\mathrm{TD}}(\mathrm{R} 1 / \mathrm{G} 2 / \mathrm{B} 2)-\mathrm{CDNA}$ thin films were 78 and $87 \%$, which corresponded to roughly 17 and $8 \%$ increments compared to the CRI of $\mathrm{QD}_{\mathrm{TD}}(\mathrm{R} 2 \mathrm{G} 1 \mathrm{~B} 1)-$ and $\mathrm{QD}_{\mathrm{TD}}(\mathrm{R} 1 \mathrm{G} 2 \mathrm{~B} 2)-\mathrm{CDNA}$ thin films, respectively.

Similar trends of EL for both mixed and stacked configurations were observed for the other R, G, and B QD concentration ratios of $1: 1: 1,2: 2: 1,1: 1: 2,1: 2: 1$, and $2: 1: 2$ (CRI values and CIE colour coordinates are shown in Table 2). This demonstrated that the reduction in $\mathrm{R}$ concentration significantly decreased the self-absorption and hence increased the CRI.

\section{Conclusions}

We prepared ligands (TOP/TBP and DDT) attached to R, G, and B QDs and DNA with CTMA (CDNA) for fabrication of QDs embedded in CDNA thin films with two distinct configurations (mixed and stacked), and measured their FTIR, PLQY, UV-vis absorbance, PL, and EL characteristics. Continuous absorption characteristics of QDs with TOP/TBP and DDT ligands $\left(\mathrm{QD}_{\mathrm{TD}}\right)$ provided large Stokes shifts and showed the benefits of single excitation via the down-conversion process. The PLQY values of $\mathrm{QD}_{\mathrm{TD}}$ in the liquid phase and in CDNA thin films were enhanced up to $95 \%$ and $25 \%$, respectively. Although the UV-vis absorbance and PL intensity of $\mathrm{QD}_{\mathrm{T}^{-}}$and $\mathrm{QD}_{\mathrm{TD}}$-CDNA thin films varied, their characteristic peak positions were not significantly altered. The dispersion of QDs in the CDNA matrix effectively stabilized the QDs, consequently leading to high PLQY. In addition, the accelerated photostability measurement revealed the stability of QD-CDNA thin films. EL spectra of the $\mathrm{QD}_{\mathrm{TD}^{-}}$ CDNA thin films with two different configurations (mixed, $\mathrm{QD}_{\mathrm{TD}}(\mathrm{R} 1 \mathrm{G} 2)-\mathrm{CDNA}$, and stacked, $\left.\mathrm{QD}_{\mathrm{TD}}(\mathrm{R} 1 / \mathrm{G} 2)-\mathrm{CDNA}\right)$ integrated on blue LEDs were obtained. Although the concentration ratio of $\mathrm{R}$ to $\mathrm{G}$ was the same, their CRI values were significantly different, 21 and $80 \%$, respectively, due to suppression of the $\mathrm{G}$ QD intensity. This indicated the self-absorption characteristics of R QDs. Consequently, the R QDs absorbed G light more in the mixed structures than in the stacked structures. Similarly, we studied white-light luminescence with a UV LED source for $\mathrm{QD}_{\mathrm{TD}}(\mathrm{RGB})-\mathrm{CDNA}$ and $\mathrm{QD}_{\mathrm{TD}}(\mathrm{R} / \mathrm{G} / \mathrm{B})-\mathrm{CDNA}$ thin films having different R, G, and B QD concentrations; these concentrations noticeably affected CRI efficiency and CIE colour coordinates. The proposed approach showed the benefits of a single excitation source due to the continuous absorbance of QDs toward higher energy and simplified RGB spectral generation of white light.

\section{Conflicts of interest}

There are no conflicts to declare. 


\section{Acknowledgements}

This work was supported by the National Research Foundation (NRF) of Korea (2012M3A6A7054855 and 2018R1A2B6008094).

\section{References}

1 T. Erdem and H. V. Demir, Nanophotonics, 2013, 2, 57-81.

2 B. Xie, R. Hu and X. Luo, J. Electron. Packag., 2016, 138, 20803-20813.

3 B. C. Fitzmorris, Y.-C. Pu, J. K. Cooper, Y.-F. Lin, Y.-J. Hsu, Y. Li and J. Z. Zhang, ACS Appl. Mater. Interfaces, 2013, 5, 2893-2900.

4 Y. Shirasaki, G. J. Supran, M. G. Bawendi and V. Bulović, Nat. Photonics, 2013, 7, 13-23.

5 E. Petryayeva, W. R. Algar and I. L. Medintz, Appl. Spectrosc., 2013, 67, 215-252.

6 J. Bao and M. G. Bawendi, Nature, 2015, 523, 67-70.

7 Y. Altıntas, S. Genc and M. Y. Talpur, Nanotechnology, 2016, 27, 295604.

8 J. C. Bonilla, F. Bozkurt, S. Ansari, N. Sozer and J. L. Kokini, Trends Food Sci. Technol., 2016, 53, 75-89.

9 S. V. Kershaw, L. Jing, X. Huang, M. Gao and A. L. Rogach, Mater. Horiz., 2017, 4, 155-205.

10 S. Ye, F. Xiao, Y. X. Pan, Y. Y. Ma and Q. Y. Zhang, Mater. Sci. Eng., $R$, 2010, 71, 1-34.

11 C. Xu and K. M. Poduska, J. Mater. Sci.: Mater. Electron., 2015, 26, 4565-4570.

12 Y.-C. Pu and Y.-J. Hsu, Nanoscale, 2014, 6, 3881.

13 J. E. Lee, E. D. Do, U. R. Lee, M. J. Cho, K. H. Kim, J.-I. Jin, D. H. Shin, S.-H. Choi and D. H. Choi, Polymer, 2008, 49, 5417-5423.

14 E. F. Schubert and J. K. Kim, Science, 2005, 308, 1274-1278.

15 U. Resch-Genger, M. Grabolle, S. Cavaliere-Jaricot, R. Nitschke and T. Nann, Nat. Methods, 2008, 5, 763-775.

16 D. Cai, X. Yuan, D. Zhu, H. Zhou, H. Li and J. Zhao, Mater. Res. Bull., 2017, 85, 222-227.

17 V. Wood and V. Bulović, Nano Rev., 2010, 1, 5202.

18 J. H. Oh, S. J. Yang and Y. R. Do, Light: Sci. Appl., 2014, 3, e141.

19 L. Zan, D. Lin, P. Zhong and G. He, Opt. Express, 2016, 24, 7643.
20 T. Erdem, S. Nizamoglu and H. V. Demir, Opt. Express, 2012, 20, 3275 .

21 S. Abe, J. J. Joos, L. IDJ Martin, Z. Hens and P. F. Smet, Light: Sci. Appl., 2017, 6, e16271.

22 A. J. Steckl, H. Spaeth, H. You, E. Gomez and J. Grote, Opt. Photonics News, 2011, 22, 34.

23 E. F. Gomez, V. Venkatraman, J. G. Grote and A. J. Steckl, Adv. Mater., 2015, 27, 7552-7562.

24 V. Arasu, S. R. Dugasani, J. Son, B. Gnapareddy, S. Jeon, J.-H. Jeong and S. H. Park, J. Phys. D: Appl. Phys., 2017, 50, 415602.

25 M. R. Kesama, S. R. Dugasani, S. Yoo, P. Chopade, B. Gnapareddy and S. H. Park, ACS Appl. Mater. Interfaces, 2016, 8, 14109-14117.

26 V. Arasu, S. R. Dugasani, M. R. Kesama, H. K. Chung and S. H. Park, Sci. Rep., 2017, 7, 11567.

27 Y. Park, S. Jeong and S. Kim, J. Photochem. Photobiol., C, 2017, 30, 51-70.

28 L. Wang, J. Yoshida, S. Sasaki, T. Kajiyama and N. Ogata, Chem. Mater., 2001, 13, 1273-1281.

29 J. Kundu, Y. Ghosh, A. M. Dennis, H. Htoon and J. A. Hollingsworth, Nano Lett., 2012, 12, 3031-3037.

30 V. Arasu, S. Jeon, J.-H. Jeong and S. H. Park, J. Mater. Chem. C, 2018, 6, 5199-5209.

31 H. Woo, J. Lim, Y. Lee, J. Sung, H. Shin, J. M. Oh, M. Choi, H. Yoon, W. K. Bae and K. Char, J. Mater. Chem. C, 2013, 1, 1983.

32 C. Yoon, T. Kim, M.-H. Shin, Y.-G. Song, K. Shin, Y.-J. Kim and K. Lee, J. Mater. Chem. C, 2015, 3, 6908.

33 S. Ananthakumar, J. Ram Kumar and S. Moorthy Babu, Mater. Sci. Semicond. Process., 2017, 67, 152-174.

34 B. D. Mistry, A Handbook of Spectroscopic Data Chemistry, Oxford Book Company, Jaipur, 2009.

35 L. S. Sohn, S. Unithrattil and W. B. Im, ACS Appl. Mater. Interfaces, 2014, 6, 5744-5748.

36 M. Zhu, X. Peng, Z. Wang, Z. Bai, B. Chen, Y. Wang, H. Hao, Z. Shao and H. Zhong, J. Mater. Chem. C, 2014, 2, 10031.

37 C.-J. Chen, C.-C. Lin, J.-Y. Lien, S.-L. Wang and R.-K. Chiang, J. Mater. Chem. C, 2015, 3, 196.

38 H. Chen, Z. Luo, R. Zhu, Q. Hong and S.-T. Wu, Opt. Express, 2015, 23, 13060. 